\title{
¿PUEDE EXISTIR UN DEBER DE SACRIFICAR LA PROPIA VIDA PARA SALVAR LA DE TERCEROS? ${ }^{1}$
}

\begin{abstract}
Federico Germán Abal
Instituto de Investigaciones Filosóficas (IIF) - Sociedad Argentina de Análisis Filosófico (SADAF) - Consejo Nacional de Investigaciones Científicas y Técnicas (CONICET)
\end{abstract}

\section{Resumen}

En un brevísimo artículo, James Sterba sostiene que existe un deber moral de sacrificar la propia vida para salvar la de terceros. Sterba fundamenta ese deber trazando una analogía con una serie de casos en los que comúnmente se aceptaría algún grado de sacrificio en beneficio de terceros. En el presente trabajo, sostengo que el argumento formulado por Sterba llega a la conclusión correcta, pero es invalido. Asimismo, señalo dos argumentos diferentes que podrían utilizarse para fundamentar la existencia de un deber de sacrificar la propia vida para salvar la de terceros.

Palabras clave: Sacrificio; Deber; Sobreexigencia; Sterba; Supererogatorio.

\section{Abstract}

In a very short article, James Sterba argues that there is a moral duty to sacrifice one's life to save the lives of others. Sterba justifies this duty by drawing an analogy with a series of cases in which some degree of sacrifice would be commonly accepted for the benefit of third parties. In this paper, I argue that Sterba's argument reaches the correct conclusion, but is invalid. In addition, I point out two different arguments to support the existence of a duty to sacrifice one's life to save the lives of others.

Keywords: Sacrifice; Duty; Demandingness; Sterba; Supererogatory.

1 [Can there be a duty to sacrifice one's life to save the lives of others? Recibido: 22/04/2020; aceptado: 01/07/2020.] 


\section{INTRODUCCIÓN ${ }^{2}$}

El sacrificio de la propia vida ha sido poco tematizado en la literatura filosófica contemporánea. Incluso dentro del debate sobre los límites de las obligaciones morales los autores suelen discutir sobre la obligatoriedad de acciones menos extremas, como la donación de órganos o de una suma importante de dinero (van Ackeren \& Archer 2018: 301).

En un brevísimo artículo, James Sterba (2013) concluye que existe un deber moral de sacrificar la propia vida para salvar la de terceros. Sterba fundamenta ese deber trazando una analogía con una serie de casos en los que comúnmente se aceptaría algún grado de sacrificio en beneficio de terceros.

Si el argumento de Sterba es convincente, entonces podríamos encontrarnos afectados por un deber moral especialmente exigente; quizás, el más exigente posible. Para algunos autores el grado de sacrificio que supone el cumplimiento de este deber (a saber, la perdida de la propia vida) podría ser una razón suficiente para rechazar el argumento de Sterba. Este rechazo suele presentarse en la literatura bajo la forma general de una objeción de la sobreexigencia.

En el presente artículo, sostengo que el argumento de Sterba llega a la conclusión correcta, pero resulta en sí mismo inaceptable. Para defender esta tesis mostraré que (1) la objeción de la sobreexigencia en su versión más extendida es errónea, (2) que el argumento de Sterba no es válido y (3) que el deber moral al que arriba en su conclusión puede defenderse de una manera diferente.

\section{RECHAZO A LA OBJECIÓN DE LA SOBREEXIGENCIA}

La objeción de la sobreexigencia puede formularse del siguiente modo (Braddock 2013: 169). ${ }^{3}$

2 Una versión previa de este trabajo fue presentada en formato de ponencia en el XIX Congreso Nacional de la Asociación Filosófica Argentina, realizado en la ciudad de Mar del Plata (Argentina). Agradezco las críticas y comentarios que las siguientes personas formularon en aquella ocasión: Santiago Armando, Daniel Busdygan, Julieta Elgarte, Cristián Fatauros, Facundo García Valverde, Eduardo Rivera López y Graciela Vidiella. También agradezco a Paola Cardozo y al Pbro. Jose Luís Puñal por su apoyo durante la producción de esta versión final. Este trabajo se realizó con el apoyo financiero de una beca doctoral del Consejo Nacional de Investigaciones Científicas y Técnicas (CONICET).

3 Las traducciones son de mi autoría. 
1. La teoría moral (o principio moral) V exige demasiado de nosotros

2. Si una teoría moral (o principio moral) exige demasiado de nosotros, entonces es incorrecta

3. Por lo tanto, la teoría moral (o principio moral) V es incorrecta

Esta objeción ha sido relevante en las últimas décadas. Varios autores consideran que el hecho de que una teoría esté expuesta a la acusación de ser sobreexigente es una prueba concluyente de su fracaso. ${ }^{4}$

Generalmente, suele considerarse al consecuencialismo como la teoría más expuesta a la objeción de la sobreexigencia (Sobel 2007). Esta vinculación se debe a la preeminencia de dicha objeción en el debate sobre justicia global, en el que se inscribe un trabajo señero de Peter Singer que sostiene la existencia de un exigente deber de beneficencia sobre la base de consideraciones consecuencialistas (van Ackeren y Kühler 2015a). En "Famine, Affluence, and Morality” (1972), Singer afirma que, si está en tu poder evitar que pase algo malo, sin tener que sacrificar nada que tenga una importancia moral comparable, entonces debes moralmente hacerlo (1972: 231).

Las implicaciones del principio de Singer en materia de ayuda humanitaria suelen considerarse radicales y muchos autores han dudado de que tal nivel de exigencia sea plausible (Cullity 2004, García Gibson 2013). No obstante, dentro del mismo debate se ha señalado que puede arribarse a conclusiones igualmente exigentes desde otras perspectivas teóricas, como el contractualismo scanloniano (Ashford 2003) o la ética de la virtud (Swanton 2009). Por su aparente carácter rigorista, también se ha calificado al deontologismo kantiano de ser sobreexigente (van Ackeren y Kühler 2015b).

Ahora bien, no es evidente qué significa que una teoría sea sobreexigente. Tal como señala Braddock, toda teoría moral plausible, al imponer algún tipo de obligación, es en cierta medida exigente (2013: 169, en la misma línea Goodin 2009: 3). Sin embargo, la idea de "sobreexigencia" remite a una pretendida obligación que va más allá de lo que puede ser razonablemente obligatorio (beyond the call of duty). J.O. Urmson en un canónico ensayo ofrece un ejemplo que ilustra esta idea.

"Podemos imaginar a un escuadrón de soldados practicando el lanzamiento de granadas de mano; una granada se desliza de la mano

4 Muestra de ello son los diferentes intentos de formular versiones del utilitarismo inmunes a esta objeción (Hooker 2000, Mulgan 2001, Norcross 2006). 
de uno de ellos y rueda por el suelo cerca del escuadrón; uno de ellos sacrifica su vida arrojándose sobre la granada y protegiendo a sus camaradas con su propio cuerpo." (1969: 63)

Urmson sostiene que el acto del soldado es valioso, pero no moralmente obligatorio y fundamenta dicha evaluación en dos características. En primer lugar, el acto no es moralmente obligatorio porque sería inapropiado que alguien exigiera al soldado que lo hiciera. En segundo lugar, el acto no es moralmente obligatorio porque el soldado no podría ser reprochado por no haberlo realizado. En palabras de Urmson, si él no hubiera actuado de ese modo, nadie podría haberle dicho "deberías haberte arrojado sobre la granada" (1969: 63).

Para Urmson, los actos moralmente obligatorios se identifican por su capacidad para ser justificadamente acompañados por ciertas actitudes reactivas de terceros (exigir y reprochar). Esta posición de raigambre strawsoniana ha sido desarrollada más recientemente por Stephen Darwall, quien sostiene que "la obligación moral y los criterios de correcto e incorrecto están conceptualmente relacionados con lo que la comunidad moral, y nosotros como miembros de ella, podemos exigir" (2006: 96). ${ }^{5}$

Este enfoque, aunque puede ser adecuado desde un punto de vista metaético, elude la respuesta que necesitamos. Si moralmente obligatorio es aquello que puede ser justificadamente exigido, entonces ¿qué puede ser justificadamente exigido?

Un modo intuitivo de responder a esta pregunta es acudiendo al costo que una acción tendría para los intereses del agente. Existe un amplio consenso respecto de que aquello que impide que una acción sea considerada obligatoria es precisamente el grado de sacrificio que conlleva (Scheffler 1994: 20, Archer 2016, McElwee 2017). Consideremos los siguientes condicionales y sus respectivas consecuencias lógicas.

1. Supererogatorio $\rightarrow$ Costo elevado

2. No Costo elevado

3. No Supererogatorio

1. Costo elevado $\rightarrow$ Supererogatorio

2. No Supererogatorio

3. No Costo elevado

5 También por Heyd (1982) y McElwee (2017). 
El primer condicional afirma que, si una acción es supererogatoria, entonces conlleva un costo elevado para el agente. Algunos autores han rechazado que esta conexión se cumpla en todos los actos supererogatorios (Horgan and Timmons 2010: 62, Ferry 2013: 579, Crisp 2013: 16). Por ejemplo, no es cierto que ayudar a un vecino desconocido con la mudanza implique necesariamente un costo elevado y, no obstante, no parece correcto afirmar que es moralmente obligatorio hacerlo.

El segundo condicional, más relevante para nuestro análisis, afirma que, si un curso de acción involucra un costo elevado, entonces es supererogatorio. Se sigue de ello que, si esa acción no es supererogatoria, entonces no involucra un costo elevado. Este condicional podría concebirse como la presentación formal de la versión más extendida de la objeción de la sobreexigencia. Si realizar X supone un costo elevado, entonces X no puede ser obligatorio. ${ }^{6}$

Sin embargo, esta afirmación es falsa. El discurso moral cotidiano reconoce la existencia de obligaciones que involucran un costo elevado para el agente. Por ejemplo, la obligación moral de saldar una deuda que hemos tomado con un familiar o un amigo se mantiene, aunque ello conlleve la imposibilidad de acudir a un concierto al que deseamos concurrir desde hace años. Si apelamos a las dos características introducidas por Urmson, es claro que el acreedor podría plausiblemente exigir al amigo o familiar deudor que cumpliera con su compromiso de pago y podría plausiblemente reprocharlo moralmente si no lo hiciera. ${ }^{7}$

6 Asimismo, se sigue del condicional que, si está prohibido realizar X (si no hacerlo no es supererogatorio), entonces no realizar X no conlleva un costo elevado. Esta consecuencia también demuestra la falsedad del condicional. Del hecho de que exista una prohibición moral de matar a un inocente para sacarle un órgano, no se sigue que no hacerlo no conlleve un costo elevado para el individuo que necesita ese órgano. Como el tratamiento habitual de la objeción de la sobreexigencia se centra en el costo de ciertas obligaciones a actuar (por ejemplo, el costo de donar un órgano, ver Sobel 2007), en este punto no me detendré en el costo de ciertas prohibiciones a actuar (por ejemplo, la prohibición de matar a un individuo inocente para extraer un órgano).

7 Goodin (2009: 1) utiliza una metáfora similar para trazar una comparación entre la exigencia de un acreedor y la de la moralidad. Solo puede decirse que el reclamo del acreedor es sobreexigente, si exige más de lo que corresponde, pero eso no puede determinarse solo apelando al costo que el pago tendría para el deudor. Ciertamente, esta afirmación es compatible con la existencia de otros deberes que podrían derrotar al deber de cumplir con el acreedor. 
Podría decirse que este tipo de casos merece un tratamiento diferenciado porque involucra una obligación especial asumida por quien solicito un préstamo hacia el prestamista. Pero precisamente ese tipo de aclaraciones son las que hacen notar que la fundamentación de la obligación moral no depende directa y exclusivamente del costo de la acción sino de otras variables, como los compromisos asumidos previamente.

No puede definirse la pretendida sobreexigencia de una acción sin acudir al contexto y al peso de las razones morales en juego. Indudablemente, puede afirmarse de una obligación moral que es exigente de manera aislada. Por ejemplo, si la deuda que un deudor debe pagar asciende a un monto muy elevado, entonces cumplir con ella es exigente independientemente de que corresponda hacerlo. Sin embargo, para determinar que esa acción es sobreexigente debe evaluarse si lo que se exige excede lo que puede ser obligatorio y para analizar esto, como resulta evidente, no basta remitirse al costo de la acción para el agente. ${ }^{8}$

El propio ejemplo de Urmson puede ilustrar un caso extremo en esta dirección. Si los miembros del pelotón hubieran asumido previamente un compromiso de dar la vida por sus compañeros, entonces el sacrificio del soldado no debería ser considerado como un acto supererogatorio, sino obligatorio. Considérese la siguiente modificación al ejemplo de Urmson que resalta este punto.

Soldado cobarde: Los miembros de un pelotón realizan al momento de incorporarse un juramento que consiste en dar la vida por sus compañeros toda vez que sea necesario. En medio de una práctica con granadas de mano, uno de los soldados deja caer accidentalmente una granada sin el seguro cerca de Federico, quien por razones de distancia es el único que podría evitar la muerte de sus compañeros lanzándose encima de la granada y conteniendo su alcance explosivo.

8 Elocuentemente, McElwee (2017: 11) introduce al debate la exigencia que Dios comanda a Abraham para que este sacrifique a su hijo Isaac (Gen. 22). Indudablemente, ese requerimiento es exigente, pero no es seguro que sea sobreexigente. Para determinar esto último, Abraham debería ponderar las razones morales de matar a su hijo y analizar si cumplir con el mandato divino excede lo moralmente obligatorio. Por supuesto, desde una teoría del divino comando, hacer lo requerido por quien es parámetro de lo moralmente obligatorio no puede exceder lo que es moralmente obligatorio. Cabe mencionar que el término "supererogatorio" fue inventado precisamente por teólogos cristianos para describir aquellas acciones que excedían positivamente lo que era comandado por Dios (Crisp 2013: 18-19, Archer 2016: 245). 
Federico decide huir y resguardarse de la explosión. La granada explota acabando con la vida de algunos de sus compañeros.

El juramento realizado por los miembros del pelotón los compele a sacrificar su vida para salvar las de sus compañeros. Por supuesto, podría ser que estuviera implícito en esa promesa que el momento esperado para dar cuenta de dicho compromiso es en el campo de batalla y no en un entrenamiento. Sin embargo, los acontecimientos se produjeron fatídicamente de ese modo. Federico no cumplió con su juramento y los soldados sobrevivientes podrían plausiblemente reprocharle el no haberlo hecho, incluso expulsarlo del pelotón. ${ }^{9}$

Nótese que la obligación moral que recae sobre Federico se sostiene, aunque fuera objetivamente cierto que en circunstancias similares los otros miembros del pelotón hubieran actuado del mismo modo que él, incumpliendo su respectivo juramento. La posibilidad conceptual de exigir cierto curso de acción y reprochar a otro individuo por actuar de manera prohibida no depende de asumir que uno actuaría fácticamente de ese modo si estuviera en condiciones similares, sino del reconocimiento de lo que es moralmente obligatorio.

Ahora bien, quienes conciben que para que una acción pueda ser justificadamente exigida el costo que la misma impone al agente no debe ser elevado, podrían sostener que esta condición no aplica a aquellos casos en los que intervienen obligaciones especiales, como promesas, juramentos o relaciones afectivas. Aunque ad hoc, este recorte es intuitivamente plausible. De hecho, el discurso moral cotidiano suele reconocer como supererogatorias mayoritariamente

9 Urmson (1969: 72), afirma lo siguiente: "Si bien no hay nada objetable en la idea de que alguien sea presionado para llevar a cabo un deber tan básico como el cumplimiento de promesas, hay algo horrible en el pensamiento de presionarlo para que realice un acto de heroísmo ... Sería un ultraje moral presionarlo para que hiciera un acto como sacrificar su vida por los demás". Urmson sostiene que el cumplimiento de promesas puede ser exigido, pero no así el sacrificio de la propia vida. Este último representaría un caso de heroísmo. Los actos heroicos son ejemplos paradigmáticos de supererogación y, por lo tanto, no son obligatorios. Urmson no contempla la posibilidad planteada por el ejemplo del Soldado cobarde, en el que el propio cumplimiento de la promesa implica el sacrificio de la propia vida. Una manera posible de concebir dicho ejemplo, que permitiría evitar una contradicción definicional (esta es, la obligación de realizar un acto heroico), es argumentando que una vez que el soldado ha asumido el compromiso de sacrificar su vida dicho sacrificio no constituye conceptualmente un acto heroico para él, sino una obligación. Ese mismo acto continúa siendo heroico (supererogatorio) para alguien que no ha asumido ese compromiso previamente, pero ya no para el soldado. Esta solución fue propuesta originalmente por Farrell (1984). 
aquellas acciones relativas a individuos con quienes no guardamos ninguna relación normativa relevante.

Retomando la crítica al principio de beneficencia defendido por Singer, es plausible pensar que una de las razones del rechazo a donar buena parte de los propios ingresos a organizaciones benéficas radica en la inexistencia de una relación normativa especial con los millones de pobres globales que serían favorecidos por nuestro aporte extraordinario. Podría ser que, en ausencia de obligaciones especiales, el costo excesivo que la acción impone al agente fuera condición suficiente para considerarla como supererogatoria. Por supuesto, la determinación del umbral en el que un costo comienza a resultar excesivo es inevitablemente arbitraria en el debate sobre deberes de beneficencia. ¿Por qué donar el 10\% de los propios ingresos sería demasiado exigente y no así el 5\%? ${ }^{10}$

Contrariamente a este tipo de casos, un curso de acción que implica el sacrificio de la propia vida es indudablemente exigente. Dentro del debate sobre la permisibilidad moral de la violencia defensiva se discute en torno a escenarios que involucran acciones cuyo resultado es, generalmente, la vida o la muerte del agente.

Parece evidente que a todo individuo le asiste un permiso para defender violentamente su vida. Sin embargo, existe un consenso respecto de que dicho permiso está sujeto a limitaciones. La mayoría de los autores coincide en que el recurso a la violencia letal en defensa de la propia vida solo puede estar justificado si (a) es necesario y si (b) no es dirigido intencionalmente contra un ser humano inocente.

Por "necesario" debe entenderse que la violencia letal es, en ese escenario, el único recurso disponible para evitar la propia muerte. Por "ser humano inocente" refiero a un individuo que no presenta ninguna amenaza y que no guarda responsabilidad moral alguna sobre la agresión que se busca evitar.

Esta segunda limitación (no dirigir intencionalmente el acto violento contra un ser humano inocente), plantea la existencia de lo que Kaufman (2010) denomina un "deber moral del martirio". Si el único

10 Tal como señala Braddock (2013: 171), los usos de la objeción de la sobrexigencia varían sobre qué conciben por sobreexigente. Por ejemplo, podría ser que una acción fuera sobreexigente por el tipo de costo que conlleva, por la cantidad del costo o por la frecuencia con que exige que se incurra en ese costo. Cualquiera sea el uso de la noción de costo que se prefiera, la objeción de la sobreexigencia se ve expuesta al mismo problema: el costo de una acción no define directa y exclusivamente su carácter obligatorio o supererogatorio. 
recurso disponible para evitar ser asesinado es matando intencionalmente a un inocente, entonces debe aceptarse la propia muerte. Considérese el siguiente ejemplo, ampliamente discutido.

Escudo humano: Un proyectil es lanzado al aire por un tornado y se dirige hacia ti. La única forma de salvar tu vida es utilizar a una persona que está observando la situación como escudo humano. Dicha persona es completamente inocente y es lo suficientemente liviana como para forzarla y protegerte del proyectil interponiendo su cuerpo. (Doggett 2011: 220)

Los autores coinciden en que utilizar a un individuo inocente como escudo humano para salvar la propia vida es impermisible (Thomson 1991, Otsuka 1994, Frowe 2014: 51). Si ese es el único medio para evitar la muerte, entonces es moralmente obligatorio sacrificarse.

Tal como la hemos estado considerando, la objeción de la sobreexigencia parece no ser procedente en este tipo de casos. Si en un escenario como el planteado en Escudo humano alguien rechazara el deber moral del martirio apelando al costo que este impondría sobre el agente, simplemente diríamos que no comprende el juego de la justificación moral. ${ }^{11}$ Pareciera que el potencial objetor estaría incurriendo en una falaz derivación del ser (lo que las personas están normalmente dispuestas a hacer) al deber ser (lo que las personas están moralmente obligadas a hacer).

Los defensores de la objeción de la sobreexigencia podrían nuevamente intentar limitar el ámbito de acciones para las cuales el costo aplica como una condición suficiente para que un acto sea supererogatorio. Por ejemplo, podrían argumentar que para aquellos actos que involucran la muerte intencional y oportunista de un inocente, el costo que no realizarlos tendría para el agente no es moralmente relevante. Aunque también resulta ad hoc, este recorte es intuitiva-

11 Podría objetarse que la alusión a un "deber moral del martirio" en Escudo humano es un exceso, ya que simplemente se hace referencia a la obligación de no matar intencionalmente a un inocente para salvar la propia vida de un evento fortuito. Sin embargo, creo que esta expresión, acuñada originalmente por Kaufman dentro del debate sobre violencia defensiva, tiene una fuerza expresiva adecuada. La idea de aceptar la propia muerte antes que incurrir en un acto inmoral guarda estrecha relación con el uso del término mártir en la tradición cristiana (por ejemplo, ver Catecismo de la Iglesia Católica 2473). Mártir es, entre otras características, aquel que no resiste su muerte, dando testimonio de una causa o convicción superior (en este caso, el cumplimiento de la obligación moral de no matar intencionalmente a un inocente). 
mente plausible. De hecho, una ligera modificación del clásico ejemplo del tranvía serviría como sustento de esta posible demarcación.

Tranvía: Te despiertas atado a una vía de tren. Un tranvía corre fuera de control por esa misma vía. El único modo de evitar que te destroce es accionando con tu pie una palanca que permitiría que el tranvía se desvíe hacia una vía paralela. En la vía paralela se encuentra atada otra persona inocente, Pedro.

Jonathan Quong sostiene que existe un permiso relativo al agente para priorizar la propia vida en este tipo de casos (2009: 533-534, también McMahan 2002: 408). En Tranvía, a diferencia de Escudo humano, la violación del derecho a la vida del individuo atado a la vía paralela no es producida de manera intencional y oportunista sino como un efecto colateral de tu acción para salvarte. Esta característica se evidencia en el hecho de que su presencia no es un medio para la acción defensiva. Si por alguna razón Pedro pudiera desatarse antes de que el tranvía cambie de vía, la acción seguiría siendo exitosa a los fines de evitar la muerte.

Ahora bien, dicho permiso se encuentra limitado por la proporcionalidad del acto defensivo en cuestión. En Tranvía, el efecto colateral negativo de accionar la palanca (esto es, la muerte de Pedro) es proporcional a su efecto intencional positivo (salvar la propia vida). Sin embargo, bastaría adicionar otra persona inocente a la vía alternativa para que la acción defensiva resultara desproporcional y, por lo tanto, que el permiso relativo al agente para priorizar la propia vida careciera de validez. La consideración que justifica ser parcial en favor de uno mismo sólo opera permisiblemente como un criterio de desempate entre casos proporcionales, pero no habilita al agente a matar de manera colateral a más de un inocente.

Antes de continuar, cabe introducir dos aclaraciones. En primer lugar, como queda evidenciado en Escudo humano, la proporcionalidad de un acto de violencia letal en defensa de la propia vida es moralmente irrelevante cuando este es dirigido de manera intencional y oportunista contra un individuo inocente. Ese tipo de acción es siempre impermisible.

En segundo lugar, es importante resaltar que la proporcionalidad es un concepto extensamente debatido en la literatura sobre violencia defensiva. En este trabajo, me limito a contemplar la proporcionalidad de actos en defensa de la propia vida y no en defensa de la de terceros inocentes. Esta distinción resulta de especial importancia, ya que suele aceptarse que los actos en defensa de la vida de terceros 
que involucran la muerte colateral de otros individuos inocentes solo son proporcionales cuando existe una significativa diferencia a favor del numero de inocentes que serán salvados. Por ello, una acción en defensa de la vida de terceros que implica la muerte colateral de un numero idéntico (o no significativamente menor) de inocentes es desproporcional y, en consecuencia, impermisible. ${ }^{12}$ Contrariamente, el permiso relativo al agente para priorizar la propia vida, potencialmente presente en contexto de autodefensa, habilita al agente a producir la muerte colateral de inocentes en caso de empate. Considérese el siguiente caso.

Francotirador: Juan está a punto de ser asesinado por un francotirador. No hay modo en que pueda esconderse de su ataque a tiempo. El único modo de evitar ser asesinado es accionando una bomba que está colocada en el piso del edificio donde está dispuesto el francotirador. Juan sabe que si detona la bomba no solo dará muerte a su agresor, sino también a varias familias que viven en ese piso.

Tal como señala Kaufman, carecemos de información sistemática respecto de las intuiciones comunes sobre casos de violencia defensiva (2010: 79). Casos como Francotirador son indudablemente difíciles, y es de esperar que las respuestas frente a estos sean divergentes. ${ }^{13}$ Sin embargo, es plausible afirmar que, si existe un deber moral del martirio frente a casos que, como Escudo humano, involucran la muerte intencional y oportunista de un inocente, existe un deber moral del martirio en aquellos escenarios en los que para defender la propia vida se debe matar colateralmente a un número mayor de inocentes. Cabe recordar que los inocentes no han realizado previamente ninguna acción que los haga moralmente responsables de una agresión injusta y no representan una amenaza. Desde su perspectiva, no hay mayor diferencia entre morir de manera intencional o colateral. En estos casos, no aplica un permiso relativo al agente para priorizar la propia vida.

Por lo tanto, si un deber moral del martirio esta plausiblemente justificado en Francotirador, entonces el intento de limitar la objeción de la sobreexigencia a casos que (a) no involucran obligaciones

12 McMahan (2017) define a este tipo de proporcionalidad, que contempla el daño colateral sobre individuos inocentes en el marco de acciones en defensa de la vida de terceros, como proporcionalidad amplia.

13 No es claro que las personas no estén dispuestas a sacrificar su vida antes que la de un tercero. Para un abordaje empírico sobre dilemas morales que involucran el autosacrificio, ver Sachdeva, Iliev, Ekhtiari y Dehghani (2015). 
especiales y (b) no involucran la muerte intencional y oportunista de inocentes, sería equivocado. Existirían otros escenarios, a saber, los que conllevan la muerte colateral de varios inocentes, en los que se mantiene una obligación moral con independencia del exigente costo que implica para el agente, a saber, la perdida de la propia vida.

Según creo, estos fallidos intentos de reducir el alcance de la objeción de la sobreexigencia evidencian que el costo de un acto no es condición suficiente para considerarlo supererogatorio, esto es, siguiendo las características de Urmson, incapaz de ser justificadamente exigido y/o reprochado. Como quedo demostrado mediante los ejemplos citados (Soldado cobarde, Escudo humano y Francotirador), existen actos moralmente obligatorios en los que un costo considerablemente alto está justificado. A diferencia de los casos habitualmente discutidos en el debate sobre deberes de beneficencia, estos ejemplos tienen la ventaja de poner a prueba a la objeción de la sobreexigencia remitiendo a escenarios que involucran un costo indudablemente alto para el agente.

\section{El ARgumento de SterbA}

James Sterba sostiene que "una de las mejores maneras de responder a cualquier pregunta moral particular es atendiendo a la respuesta que daríamos a preguntas morales relacionadas" (2013: 108).

Siguiendo esta estrategia, Sterba se pregunta si existe una obligación moral de frustrar un plan para hacer estallar una bomba que destruiría una ciudad a costa de la propia vida trazando una analogía con el modo en que responderíamos a preguntas similares respecto de la existencia de otras obligaciones de hacer sacrificios en beneficio de terceros.

Por ejemplo, afirma Sterba, suele aceptarse que existe una obligación de realizar sacrificios económicos por los pobres y necesitados. El autor sostiene que esa obligación es bastante exigente, alcanza a los pobres de países distantes y a futuras generaciones, y lleva a un compromiso igualitario de usar solamente la cantidad de recursos necesaria para satisfacer nuestras necesidades básicas. Cualquier uso por encima de ese umbral mínimo implica privar a los pobres globales y a las futuras generaciones de los recursos que necesitan para satisfacer sus propias necesidades básicas, lo que representa una violación de su derecho al bienestar (2013: 108). 
Sterba también afirma que se reconoce ampliamente la existencia de una obligación de arriesgar la propia vida combatiendo por nuestro país en el marco de una guerra justa. Asimismo, existen situaciones en las que se acepta que la vida de un individuo puede ser sacrificada para salvar a terceros, a saber, en casos como el del tranvía que plantea la necesidad de elegir entre matar a un individuo o dejar morir a cinco.

Finalmente, Sterba considera dos versiones de un ejemplo presentado originalmente por Philippa Foot. El ejemplo plantea un escenario en el que la única manera de evitar que un grupo de personas mueran ahogadas dentro de una cueva es haciendo estallar el cuerpo de un individuo que obstruye la salida. En la primera versión, el obstructor moriría ahogado junto con el resto de las personas de no producirse la explosión. En la segunda versión, el obstructor mantiene la cabeza fuera de la cueva y no moriría ahogado.

Sterba afirma que en la primera versión nadie negaría la permisibilidad de hacer estallar el cuerpo del obstructor, quien en cualquier escenario moriría. En la segunda versión, dado que el obstructor no moriría ahogado, la permisibilidad moral del acto de matarlo es materia de discusión. Sin embargo, Sterba afirma razonablemente que buena parte de la discusión sobre la permisibilidad moral de matar al obstructor inocente radica en la cantidad de personas inocentes que podrían ser rescatadas. Ciertamente, es plausible afirmar que la clásica distinción entre matar y dejar morir pierde su potencia intuitiva cuando aumenta el número de inocentes que perecen por nuestra decisión de no cometer un acto prima facie inmoral.

De estos ejemplos que se presumen análogos, Sterba extrae como conclusión que "claramente" existe una obligación moral de sacrificar la propia vida para evitar la explosión de la bomba que destruiría la ciudad (2013: 109).

Detengámonos en la metodología escogida por Sterba. Como es sabido, el uso de comparaciones entre casos análogos fue popularizado por Singer (1972). Garrett Cullity denominó a la estrategia empleada por Singer como "modelo de subsunción" (2004: 12-14). Este modelo avanza siguiendo tres pasos.

El primer paso consiste en identificar uno o varios juicios morales particulares e intuitivos sobre ciertos casos en los que tenemos gran confianza. Esto significa que mantienen su potencia intuitiva tras un proceso reflexivo que busca alertar sobre posibles sesgos o errores. El segundo paso consiste en postular un principio que sería la me- 
jor explicación de esos juicios morales considerados. El tercer paso consiste en aplicar el principio generado en el paso previo a casos análogos sobre los cuales no tenemos juicios morales confiables o intuiciones demasiado claras. Dicho principio iluminaría el caso dudoso, manteniendo la coherencia con otras intuiciones y principios.

El modelo de subsunción puede concebirse como una parte del funcionamiento del método del equilibrio reflexivo. El rasgo característicamente coherentista del equilibrio reflexivo es que ningún componente del proceso se justifica por sí solo, con independencia del propio mecanismo de ajuste continuo y de búsqueda de equilibrio. Así, incluso los juicios particulares que merecen nuestra mayor confianza pueden ser sujetos a revisión a la luz de otros juicios y principios de mayor alcance explicativo.

Indudablemente, ciertas creencias presentan una fuerza especial en relación a otras y el costo conceptual de revisarlas es demasiado alto. Esto ha llevado a algunos autores a concebir el equilibrio reflexivo como una forma de "fundacionismo modesto", en el que ciertas creencias tienen un status epistémico privilegiado independientemente de su coherencia con otras creencias, sin ser por ello inmunes a revisión (McMahan 2000, Sandberg \& Juth 2011, de Maagt 2016: 14). El primer paso del modelo de subsunción remite al reconocimiento de juicios morales considerados que operan como fundamento modesto a partir del cual puede avanzarse en la justificación de principios relativos a una dimensión práctica específica.

Esta versión del equilibrio reflexivo no está exenta de problemas. Una consideración detallada sobre estas complicaciones excedería los límites del presente trabajo. Dada su preeminencia dentro de la literatura filosófica analítica contemporánea, asumo este fundacionismo modesto como la metodología adecuada desde la cual argumentar. ${ }^{14}$

Ahora bien, para poder trazar una analogía entre dos escenarios diferentes, tal como establece el tercer paso del modelo de subsunción, y extraer conclusiones normativas de uno hacia otro se requiere

14 Tal como señala de Maagt (2016), esta versión del equilibrio reflexivo en términos de "fundacionismo modesto" conduce a un callejón sin salida. O bien reconoce la fuerza epistémica independiente de ciertas creencias básicas (juicios particulares considerados o intuiciones), comprometiéndose con alguna variante de intuicionismo, a costa de perder su carácter propiamente coherentista, o bien enfatiza la posibilidad de revisar esas creencias, a costa de renunciar a cualquier intento de lograr objetividad moral. 
la mediación de uno o varios principios que recojan todas las variables que se asumen como moralmente relevantes en dichos escenarios. De otro modo, podría objetarse que se ha establecido una falsa analogía entre casos que guardan características normativas diferentes.

El problema del argumento de Sterba es que no presenta ningún principio que permita mediar entre escenarios notablemente diferentes entre sí. No hay ninguna razón para creer que de la confianza que presentan los juicios particulares que Sterba introduce (acerca de las obligaciones para con los pobres globales, de participar en una guerra justa, del ejemplo del tranvía y del obstructor), se siga "claramente" (sic) la obligación de sacrificar la vida para evitar la explosión de una bomba que destruirá la ciudad. Ninguno de los deberes considerados por Sterba incluye la propia muerte y se requiere alguna mediación conceptual plausible para llegar desde estos a un deber especialmente exigente como el de autosacrificio.

Podría decirse que el deber de participar en una guerra justa, cuyo carácter obligatorio cabría objetar per se, se acerca bastante al deber de sacrificar la propia vida en beneficio de terceros y que, por lo tanto, de existir el primero podría justificarse el segundo. Sin embargo, existen variables moralmente relevantes para el establecimiento de un deber de participar en una guerra justa que no se replican en el escenario de la destrucción de la ciudad que podría evitarse mediante el sacrificio de la propia vida. Estas diferencias, según creo, hacen que la comparación entre ambos ejemplos constituya una falsa analogía.

En primer lugar, el deber de participar en, al menos, algunas guerras justas, puede reconocerse como un tipo específico de deber cívico asumido por todos los ciudadanos como contrapartida de los beneficios de formar parte de una determinada comunidad política. Ningún compromiso análogo puede encontrarse en el ejemplo de la bomba presentado por Sterba. En este sentido, una comunidad política puede exigir a ciertos ciudadanos que se dispongan a combatir en su defensa y reprocharlos moralmente si no lo hacen, pero no puede exigir a cada uno de ellos individualmente que sacrifiquen su vida para salvar a otros compatriotas de un atentado terrorista ni reprocharlos si se niegan. Al menos, no sobre la base de un compromiso cívico previo, que permite establecer, en parte, el deber de participar en una guerra justa. 
En segundo lugar, participar en una guerra, aunque supone un riesgo considerable de morir, no implica una muerte segura. A modo de ejemplo, puede apelarse a las terribles cifras de la Segunda Guerra Mundial. Dentro de esta se destaca la batalla de Stalingrado, considerada la más sangrienta de la historia. Al momento de la contraofensiva soviética, el ejército rojo, que resultó ser el bando más diezmado de la contienda, contaba con 1.143.500 soldados ubicados en Stalingrado, de los cuales murieron (o desaparecieron) 154.885 y resultaron heridos 330.892 (Glantz \& House 1995). Estas cifras permiten establecer que cada soldado soviético tenía un 13,55\% de probabilidad de morir en combate y un 30\% de terminar herido (asumiendo que formaban parte del destacamento ubicado en Stalingrado). Por supuesto, estos porcentajes disminuyen en todas las otras batallas conocidas. Considero que existe una diferencia normativa relevante entre el deber de participar de un conflicto bélico que, en el peor de los casos conocidos, conlleva un 13,55\% de morir y el deber de realizar una acción que implica una muerte segura. Las consideraciones morales que justificarían el primero no permitirían, en ausencia de un argumento plausible, justificar el segundo.

Del mismo modo, podría señalarse que, de los ejemplos del tranvía y del obstructor, que establecen la permisibilidad de matar a un individuo inocente para salvar a muchos (de manera colateral y de manera intencional, respectivamente), se sigue un deber de sacrificarnos nosotros mismos para salvar a ese mismo numero de personas. Aunque se aceptara que los ejemplos del tranvía y del obstructor dan cuenta de un deber de matar a la persona inocente y no tan solo de un permiso para hacerlo, de ello no se sigue, en ausencia de un argumento plausible, que exista un deber de autosacrificio.

A simple vista, es consistente que un individuo se muestre dispuesto a aceptar la fuerza intuitiva de estos casos y, al mismo tiempo, niegue la existencia de una obligación de sacrificarse por terceros o de no resistir su muerte por parte de quienes intentan salvar a muchos.

El argumento de Sterba recoge la obligación de hacer ciertos sacrificios por otras personas, lo que resulta fácilmente aceptable, pero no prueba que estemos obligados a dar la vida por ellas.

Aunque el argumento de Sterba es invalido, ya que de sus premisas no se sigue la conclusión pretendida, es posible apelar al mismo modelo de subsunción y formular dos principios que permitirían extraer conclusiones de juicios particulares intuitivos hacia un escena- 
rio en el que se presenta la alternativa de salvar a terceros sacrificando la propia vida. Dicho de otro modo, es posible dotar al argumento de Sterba de los principios que carece.

\section{iV. Criterio de Superioridad de Pareto}

En primer lugar, consideremos la primera versión del ejemplo de Foot mencionado por Sterba. En ese caso parece claro que existe la opción permisible de matar al obstructor, dado que moriría de todos modos junto al resto de las personas dentro de la cueva. Tal como demuestra la contraposición con la segunda versión del ejemplo, la relevancia moral del hecho de que el obstructor morirá en cualquiera de los escenarios futuros posibles es determinante. Un modo de fundamentar este juicio es acudiendo al Criterio de Superioridad de Pareto. ${ }^{15}$

De acuerdo con este criterio, una situación es Pareto superior si en relación a otras alternativas disponibles ninguno de los involucrados se encuentra en una posición peor y al menos uno de ellos se encuentra en una posición mejor (Farrell 2015: 83-84). Este criterio es considerado una exigencia de la racionalidad; no habría ninguna razón atendible para negarse a elegir aquel escenario que mejora la posición de alguno(s) de los involucrados y no perjudica, en ningún sentido relevante, a ninguno de ellos. ${ }^{16}$

La fuerza intuitiva del Criterio de Superioridad de Pareto en términos morales radica en que los beneficios de unos no se obtienen a partir de los sacrificios que otros podrían evitar. La aceptación del criterio como explicación del juicio particular sobre la primera versión del ejemplo de Foot, debería llevarnos a la obligación de sacrificar nuestra propia vida si estuviéramos en la posición del obstructor.

Dicha obligación moral se justifica en la plausible prohibición a negarse a beneficiar significativamente a terceros cuando ello no

15 Deriva su nombre del sociólogo y economista italiano Wilfredo Pareto (1848 - 1923).

16 Puede haber situaciones Pareto superiores en un sentido restringido, que acarrean perjuicios para algunos en otra dimensión. Por ejemplo, si una situación B no empeora a ninguno de los involucrados en términos económicos, pero mejora enormemente la situación económica de algunos pocos, podrían producirse consecuencias indeseables producto de la desigualdad de ingresos resultante (por ejemplo, los mejor situados podría tener una influencia mayor en la toma de decisiones políticas). 
conlleva ningún costo para uno mismo, ya que no existe escenario alternativo en que podamos evitar nuestra propia muerte. Cualquier consideración mínimamente razonable sobre el bienestar de terceros debería comprometernos con esta decisión extrema.

Nótese que esta obligación puede convertirse en un deber moral del martirio simplemente redescribiendo el escenario de modo en que la única manera de lograr la situación Pareto superior es no impidiendo el propio asesinato a manos de un tercero (por ejemplo, por parte de alguno de los individuos atrapados en la cueva).

No obstante, esta defensa del Criterio de Superioridad de Pareto podría ser objeto de rechazo. El clásico experimento de Jim y los indios, formulado originalmente por Bernard Williams, puede concebirse como un contraejemplo de este criterio. Como es sabido, el ejemplo consiste en un individuo, Jim, que en el marco de una expedición se encuentra con una veintena de indios puestos contra un muro quienes, tras ser seleccionados de manera azarosa, están a punto de ser fusilados por un pelotón con el objetivo de infundir temor en la población y desincentivar potenciales protestas contra el gobierno de ese país. El capitán al mando del pelotón, Pedro, advierte la presencia de Jim y, luego de interrogarlo y reconocerlo como un visitante honorable de otro país, decide ofrecerle la posibilidad de matar él mismo a uno de los indios. Si acepta, entonces, como nota especial de la ocasión, los otros indios serán puestos en libertad. Si rehúsa, Pedro hará lo que iba a hacer previamente y matará a los veinte indios (1981: 108-109).

Ante esta propuesta, el Criterio de Superioridad de Pareto exige a Jim que opte por matar a uno de los indios y evite la muerte de los otros 19 a manos de Pedro y su pelotón. Esto se debe al hecho de que el beneficio obtenido por los 19 sobrevivientes no se obtiene a partir de un sacrificio que el indio seleccionado podría evitar si Jim decidiera actuar de otro modo.

Según creo, la aplicación del criterio nos lleva, del mismo modo que lo hace en el ejemplo de Foot, al resultado moralmente correcto. Sin embargo, entiendo que este ejemplo puede motivar a los lectores a rechazarlo y, en consecuencia, merece especial atención.

Cabe comenzar aclarando que Williams introduce este experimento, en el marco de un brillante ensayo crítico del utilitarismo, con el objetivo de ilustrar la idea de responsabilidad negativa que los consecuencialistas están comprometidos a aceptar. Dado que el consecuencialismo atribuye valores a los estados de cosas, como agen- 
tes morales somos responsables tanto de las cosas que, en el sentido cotidiano más estricto, producimos, como lo somos de aquellas cosas que podemos o dejamos de prevenir (1981: 105). La noción de responsabilidad negativa recoge este ultimo tipo de responsabilidad (sobre aquellas consecuencias que podemos o dejamos de prevenir).

Williams considera que está noción borra una distinción normativamente relevante entre lo que cada uno de nosotros hace, y por lo que cada uno es especialmente responsable, y lo que los otros hacen, aun cuando podemos evitarlo. El ejemplo de Jim busca ilustrar esta distinción. Jim no es responsable positivamente de lo que Pedro hará si él se niega a matar a uno de los indios. Contrariamente, si acepta su propuesta, será responsable positivamente de cometer un acto injusto, a saber, la muerte de un inocente. Sin embargo, cabe preguntarse si está distinción basta para justificar una decisión opuesta a la que exigiría el Criterio de Superioridad de Pareto en este escenario. El propio Williams parece opinar que en la situación de Jim la decisión de matar a uno de los indios para evitar la muerte de 19 es "obviamente" correcta (1981: 109). Hasta donde alcanzo a ver, dicha “obvia” corrección se debe a las siguientes razones.

En primer lugar, aun cuando la distinción entre responsabilidad negativa y responsabilidad positiva sea conceptualmente atendible, de ello no parece concluirse que la responsabilidad negativa sea moralmente irrelevante. Considérese el clásico ejemplo del niño ahogándose en la fuente, del que Singer extrae conclusiones radicales en materia de deberes de beneficencia. No es aceptable que un individuo en condiciones de rescatar al niño niegue la existencia de un deber de hacerlo porque no ha formado parte de la cadena causal de acontecimientos que llevaron al niño a esa situación. La mayor parte de los deberes de rescate parten de reconocer la relevancia moral de la responsabilidad negativa sobre ciertas consecuencias que pueden prevenirse.

En segundo lugar, considero que esta relevancia no se ve afectada por la participación de un tercer agente positivamente responsable en la cadena causal que produce las consecuencias indeseables. Williams concibe la idea de responsabilidad negativa en un sentido restringido. En este sentido, el uso problemático de dicha idea consistiría exclusivamente en su predicación respecto de las consecuencias de acciones que realizan otros agentes positivamente responsables y que podrían prevenirse. Ese es, precisamente, el caso de Jim, quien al negarse a matar sería responsable negativamente por las conse- 
cuencias del accionar de un tercer agente positivamente responsable, a saber, Pedro. Sin embargo, incluso esta versión restringida de la idea de responsabilidad negativa no permite afirmar que esta sea moralmente irrelevante. Considérese el ejemplo del tranvía. El hecho de que el tranvía haya sido puesto en funcionamiento por un villano que previamente ató a los inocentes a las vías no altera el deber moral de redireccionarlo, que cualquier individuo tendría frente a esa misma situación en ausencia del villano.

En tercer lugar, negarse a realizar una acción que salvará la vida de 19 personas para no cometer un acto prima facie inmoral constituye un ejemplo de purismo moral inaceptable, que en el caso de Jim se preserva a costa de la vida de personas inocentes. Ciertamente, Jim no es igualmente responsable que Pedro por las consecuencias que se seguirán de esa decisión. Sin embargo, reconocer esa diferencia no exime a Jim del deber de aplicar el Criterio de Superioridad de Pareto y de ser reprochado moralmente si decide no hacerlo. Williams afirma en el ejemplo que "los hombres condenados, y los vecinos del pueblo, conocen la situación, y le suplican (a Jim) obviamente que acepte (la propuesta de Pedro)" (1981: 109). Ellos serían los sujetos de las actitudes reactivas (de exigencia y de reproche) que, como vimos en la sección II, acompañan a las obligaciones morales.

Finalmente, si se acepta que la clásica distinción entre matar y dejar morir pierde su potencia intuitiva al aumentar el número de inocentes que perecen por nuestra decisión de no cometer un acto prima facie inmoral, entonces debe aceptarse el acto de matar máxime si no impone al fallecido un daño que podría haberse evitado de otro modo.

Estas razones permiten afirmar que la responsabilidad negativa por las consecuencias que podrían evitarse si aplicáramos el Criterio de Superioridad de Pareto es moralmente relevante y nos exige sacrificar la propia vida cuando ello produciría una situación Pareto Superior. ${ }^{17}$

\section{Transitividad}

Podría decirse razonablemente que los casos en los que se plantea la posibilidad de aplicar el Criterio de Superioridad de Pareto son ex-

17 Esta sección fue enormemente beneficiada por los comentarios de un revisor anónimo de Télos. 
traños. La mayoría de los ejemplos discutidos suponen que los beneficios de unos se logran mediante el sacrificio evitable de, al menos, un tercero. Este es precisamente el caso del ejemplo que le interesa discutir a Sterba, en el que el sacrificio de la propia vida permite evitar la explosión de una bomba que destruirá la ciudad.

Considero que existe una obligación de sacrificarse para salvar a un cierto número de inocentes. Para probar esto basta aplicar el Axioma de Transitividad que nos permite extraer conclusiones normativas de juicios considerados.

Como señalé en la sección II, existe un amplio consenso respecto de la prohibición de usar a un tercero inocente como escudo humano. Matar a una persona intencionalmente de ese modo implica una inaceptable vulneración de su derecho a no ser dañado. Asimismo, es plausible afirmar que existe una prerrogativa en favor de matar colateralmente a un tercero inocente para salvar la propia vida en un caso como Tranvía. Aunque ambas acciones producen el mismo resultado, a saber, la muerte de la persona inocente, merecen evaluaciones morales diferentes. Esta diferencia radica en el modo en que se produce dicha muerte. En Escudo humano, la víctima es asesinada de manera intencional y su muerte es utilizada de manera oportunista como un medio para evitar la propia. En Tranvía, la víctima muere de manera colateral y no es utilizada como un medio; de hecho, si la vía alternativa estuviera vacía, la acción de redireccionar el tranvía sería igualmente efectiva a los fines de evitar la propia muerte.

Estas afirmaciones ampliamente aceptadas podrían presentarse del siguiente modo.

1. No matar a P intencionalmente y de manera oportunista (escudo humano) > No matar a P colateralmente (tranvía)

El símbolo “>” expresa una relación de importancia moral. En 1, se expresa que no matar a un inocente (P) en un caso como Escudo humano es más importante moralmente que no matarlo en un caso como Tranvía. La relación de "ser moralmente más importante" no debería parecer misteriosa. Simplemente expresa la fuerza normativa especial de ciertas obligaciones/prohibiciones en relación a otras. Así, la prohibición de matar a $\mathrm{P}$ en Escudo humano es más importante moralmente que la prohibición de matarlo en Tranvía. Esto se evidencia en el hecho de que la segunda prohibición puede ser derro- 
tada mediante la prerrogativa de salvar la propia vida, mientras que la primera no admite esa posibilidad.

2. No matar a P intencionalmente y de manera oportunista (escudo humano) $>$ Salvar mi vida

Dentro de Escudo humano puede establecerse una relación de importancia moral entre la prohibición de matar a $\mathrm{P}$ y la acción de salvar la propia vida. En otras palabras, es más importante moralmente cumplir con la prohibición de matar a P que salvarse.

Ahora bien, la prohibición de matar a un individuo inocente de manera intencional y oportunista no es absoluta. Es posible imaginar un escenario en el que las consecuencias a evitar son lo suficientemente graves como para justificar la violación de dicha prohibición. Esto puede afirmarse atendiendo a juicios considerados comúnmente aceptados. Por ejemplo, existe un amplio consenso sobre la obligatoriedad de redireccionar el tranvía y matar colateralmente a un individuo inocente cuando ello permitiría salvar a 5 inocentes. Ese curso de acción parece estar justificadamente acompañado de actitudes reactivas, de reproche y de exigencia, por parte de las personas que morirían de no redireccionarse el tranvía. Esta afirmación puede expresarse del siguiente modo.

3. Salvar a 5 inocentes (tranvía) > No matar a P colateralmente (tranvía)

Pero debe existir alguna instancia en que la prohibición de matar a un individuo inocente de manera intencional y oportunista sea menos importante moralmente que la prohibición de matar a $n$ cantidad de individuos inocentes de manera colateral.

Si esto no fuera verdadero, entonces sería obligatorio matar colateralmente a cualquier número de individuos inocentes para no matar de manera intencional y oportunista a un individuo inocente. Esta conclusión parece inaceptable. Los inocentes no han realizado ninguna acción que los lleve a perder su derecho a no ser dañados y desde su perspectiva es indistinto morir de manera colateral o intencional. Aunque la distinción intencional/colateral es relevante normativamente y expresa diferencias en el modo en que tratamos a terceros, no puede fundar una diferencia absoluta que nos obligue a evitar matar intencionalmente a un inocente mediante la muerte 
colateral de muchos otros inocentes. Esta relación puede expresarse del siguiente modo.

4. No matar a n inocentes colateralmente $>$ No matar a P intencionalmente y de manera oportunista

Ahora bien, conceptualmente, debe existir alguna instancia en que la obligación de no matar colateralmente a $n$ inocentes, establecida en 4, sea menos importante moralmente que salvar a $n+x$ inocentes. Esta conclusión puede establecerse en virtud de 3 , que establece la existencia de una instancia en que la prohibición de matar colateralmente a un inocente $(\mathrm{P})$ es menos importante moralmente que salvar a 5 inocentes. Como señalé anteriormente, la distinción entre matar/dejar morir tiene una fuerza intuitiva que parece reducirse cuando el número de personas inocentes que dejaríamos morir asciende significativamente. En esas circunstancias, puede afirmarse la existencia de una obligación de violar la prohibición de matar a $n$ inocentes colateralmente.

5. Salvar a $n+x$ inocentes $>$ No matar a $n$ inocentes colateralmente

Aplicando el axioma de transitividad, de 4 y 5 se sigue que salvar a $x$ $+n$ inocentes es más importante moralmente que la obligación de no matar a $\mathrm{P}$ intencionalmente y de manera oportunista.

6. Salvar a $n+x$ inocentes $>$ No matar a $\mathrm{P}$ intencionalmente y de manera oportunista

Si esto es así, aplicando nuevamente el axioma de transitividad, se sigue de 2 y 6 que salvar a $n+x$ inocentes es más importante moralmente que salvar mi propia vida.

7. Salvar a $n+x$ inocentes $>$ Salvar mi vida

Esta afirmación establece la importancia moral superior de salvar a $n$ $+x$ inocentes respecto de salvar la propia vida. Esto significa que de plantearse un escenario en el que debemos optar entre un curso de acción $\mathrm{A}$, que conlleva la muerte de $n+x$ inocentes, y un curso de acción $\mathrm{B}$, que conlleva la propia muerte, debe optarse por este último.

El axioma de transitividad nos compromete a sacrificar la vida toda vez que es necesario para salvar a un número significativo de 
inocentes $(n+x)$. La misma cifra que nos obligaría a matar intencional y de manera oportunista a un inocente $(\mathrm{P})$.

\section{Conclusión}

En este trabajo he mostrado que el argumento de Sterba debe rechazarse, no porque su conclusión establezca la existencia de un deber sobreexigente sino porque no presenta ningún principio que permita fundamentar la obligación especifica de sacrificar la propia vida a partir de juicios intuitivos en los que se acepta otro tipo de sacrificios. El deber de autosacrificio para salvar a terceros es normativamente diferente a los deberes incluidos por Sterba en sus premisas.

Sin embargo, es posible formular dos argumentos en defensa de la existencia de dicho deber. El primero de ellos parte del ejemplo de Foot presentado por Sterba y fundamenta un deber de sacrificar la propia vida en aquellos escenarios donde hacerlo sería Pareto Superior. El segundo argumento parte de una serie de juicios intuitivos sobre la relación de importancia moral entre obligaciones/prohibiciones y establece, mediante la aplicación del Axioma de Transitividad, que existe un deber de sacrificar la propia vida cuando hacerlo permite salvar la vida de un numero $n+x$ de inocentes, donde $n+x$ es la cantidad de inocentes que haría permisible el asesinato intencional y oportunista de un individuo inocente.

Me permito concluir que la pregunta que da título al presente trabajo debe responderse afirmativamente pero no por las razones esgrimidas por Sterba.

\section{BIBLIOGRAFÍA}

Ackeren, Marcel van \& Kühler, Michael: "Ethics on Edge? Moral Demandingness and Ought Implies Can”. Preprints and Working Papers of the Centre for Advanced Study in Bioethics, (2015a). <http://dx.doi. org/10.4324/9781315740812-1>

Ackeren, Marcel van \& Kühler, Michael: "Kant and Moral Demandingness". Ethical Theory and Moral Practice, 18, (2015b), págs. 75-89. <http://dx.doi. org/10.1007/s10677-014-9510-3>

Ackeren, Marcel van \& Archer, Alfred: "Self-Sacrifice and Moral Philosophy". International Journal of Philosophical Studies, Vol. 26, No. 3, (2018), págs. 1-7. <http://dx.doi.org/10.1080/09672559.2018.1489638>

Archer, Alfred: "Supererogation, Sacrifice, and the Limits of Duty". The Southern Journal of Philosophy, Vol. 54, Issue 3, (2016), págs. 333-354. <http:// 
dx.doi.org/10.1111/sjp.12176>

Ashford, Elizabeth: "The Demandingness of Scanlon's Contractualism". Ethics, Vol. 113, No. 2, January, (2003), págs. 273-304. <http://dx.doi. org/10.1086/342853>

Braddock, Matthew: "Defusing the demandingness objection". Journal of Social Philosophy, Vol. 44, No. 2, Summer, (2013), págs. 169-191.

Crisp, Roger: "Supererogation and Virtue". Oxford Studies in Normative Ethics, Vol. 3, (2013), págs. 13-34. <http://dx.doi.org/10.1093/acprof:oso/9780199685905.003.0002>

Cullity, Garrett: The Moral Demands of Affluence. Oxford, Clarendon UP, 2004. <http://dx.doi.org/10.1093/0199258112.001.0001>

Darwall, Stephen: The Second Person Standpoint. Cambridge, Harvard UP, 2006.

De Maagt, Sem: "Reflective Equilibrium and Moral Objectivity". Inquiry, Vol. 6o, No. 5, (2017), págs. 443-465. <http://dx.doi.org/10.1080/002017 4X.2016.1175377>

Doggett, Tyler: "Recent Work on the Ethics of Self-Defense". Philosophy Compass, Vol. 6, Issue 4, April, (2011), págs. 220-233. <http://dx.doi.org/10.1111 /j.1747-9991.2010.00382.x>

Farrell, Martín: “Supererogación y Deber”. Análisis Filosófico, Vol. 4, No. 1, (1984), págs. 37-43.

Farrell, Martín: Enseñando Ética. Buenos Aires, Editorial Universidad de Palermo, 2015.

Ferry, Michael: "Does Morality Demand Our Very Best? Moral Prescriptions and the Line of Duty”. Philosophical Studies, Vol. 165, Issue 2, (2013), págs. 573-589. <http://dx.doi.org/10.1007/s11098-012-9968-6>

Frowe, Helen: Defensive Killing. Oxford, Oxford UP, 2014. <http://dx.doi. org/10.1093/acprof:oso/9780199609857.001.0001>

García Gibson, Francisco: “¿Cuánto debemos donar a las agencias humanitarias?: Defensa de una posición moderada”. Revista Latinoamericana de Filosofía, Vol. 39, Issue 2, (2013), págs. 245-271.

Glantz, David \& House, Jonathan: When Titans Clashed: How the Red Army Stopped Hitler. Lawrence, University Press of Kansas, 1995.

Goodin, Robert: "Demandingness as a virtue". The Journal of Ethics, Vol. 13, No. 1, (2009), págs. 1-13. <http://dx.doi.org/10.1007/s10892-007-9025-4>

Heyd, David: Supererogation: Its Status in Ethical Theory. Cambridge, Cambridge UP, 1982.

Heyd, David: "Supererogation". En The Stanford Encyclopedia of Philosophy. Zalta, Edward (ed.), 2019, <https://plato.stanford.edu/archives/win2019/ entries/supererogation/>

Hooker, Brad: Ideal Code, Real World. Oxford, Oxford UP, 2000. 
Horgan, Terry \& Timmons, Mark: "Untying a Knot from the Inside Out: Reflections on the Paradox of Supererogation". Social Philosophy and Policy, 27, (2010), págs. 29-63.

Kaufman, Whitley: "Self-defense, Innocent Aggressors, and the Duty of Martyrdom”. Pacific Philosophical Quarterly, 91, (2010), págs. 78-96. <http:// dx.doi.org/10.1111/j.1468-0114.2009.01359.x>

McElwee, Brian: "Demandingness objections in Ethics". The Philosophical Quarterly, Vol. 67, Issue 266, January, (2017), págs. 84-105. <http://dx.doi. org/10.1093/pq/pqw020>

McMahan, Jefferson: “Moral Intuition”. En The Blackwell Guide to Ethical Theory. LaFollete, Hugh (ed.), Chichester, Blackwell, 2000.

McMahan, Jefferson: The Ethics of Killing: Problems at the Margins of Life. Oxford, Oxford UP, 2002.

McMahan, Jefferson: “Liability, Proportionality, and the Number of Aggressors”. En The Ethics of War. Bazargan, Saba and Rickless, Samuel (eds.), New York, Oxford UP, 2017. <DOI:10.1093/acprof:oso/9780199376148.003.0001>

Mulgan, Tim: The Demands of Consequentialism. Oxford, Oxford UP, 2001.

Norcross, Alastair: "Reasons without Demands: Rethinking Rightness". En Contemporary Debates in Moral Theory. Dreier, James (ed.), Malden MA, Blackwell, 2006.

Otsuka, Michael: "Killing the Innocent in Self-Defense". Philosophy and Public Affairs, Vol. 23, No. 1, (1994), págs. 74-94. <http://dx.doi.org/10.1111/j.1088-4963.1994.tboooo5.x>

Quong, Jonathan: "Killing in self-defense”. Ethics, 119, (2009), págs. 507-537. $<$ http://dx.doi.org/10.1086/597595>

Singer, Peter: "Famine, Affluence, and Morality". Philosophy and Public Affairs, 1, No. 2, Spring, (1972), págs. 229-243. <http://dx.doi. org/10.4324/9781315254210-1>

Sachdeva, S., Iliev, R., Ekhtiari, H. \& Dehghani, M.: "The Role of Self-Sacrifice in Moral Dilemmas”. PLoS ONE, 10 (6), (2015). <http://dx.doi.org/10.1371/ journal.pone.0127409>

Sandberg, Joakim \& Juth, Niklas: "Ethics and Intuitions: A Reply to Singer". The Journal of Ethics, Vol. 15, No. 3, September, (2011), págs. 209-226. <https://doi.org/10.1007/s10892-010-9088-5>

Scheffler, Samuel: The Rejection of Consequentialism. Oxford, Oxford UP, 1994. <http://dx.doi.org/10.1093/0198235119.001.0001>

Sobel, David: "The Impotence of the Demandingness Objection". Philosophers Imprint, Vol. 7, No. 8, September, (2007). <http://dx.doi.org/10.1093/ acprof:oso/9780198712640.003.0013>

Sterba, James: “A Moral Obligation to Sacrifice Our Lives?”. Journal of Social Philosophy, Vol. 44, No. 2, (2013), págs. 108-109. <http://dx.doi.org/10.1111/ 
josp.12015>

Swanton, Christine: "Virtue Ethics and the Problem of Demandingness". En The Problem of Moral Demandingness. Chappell, Tim (ed.), London, Palgrave, 2009.

Thomson, Judith Jarvis: "Self-Defense". Philosophy and Public Affairs, Vol. 20, No. 4, (1991), págs. 283-310

Urmson, James Opie: "Saints and Heroes". En Moral Concepts. Feinberg, Joel (ed.), Oxford, Oxford UP, 1969.

Williams, Bernard: "Una crítica del Utilitarismo". En Utilitarismo: Pro y Contra. Smart, John Jamieson Carswell \& Williams, Bernard (eds.), Madrid, Tecnos, 1981.

Federico Germán Abal

Instituto de Investigaciones Filosóficas (IIF)

Sociedad Argentina de Análisis Filosófico (SADAF)

Consejo Nacional de Investigaciones Científicas y Técnicas (CONICET) e-mail: <federicogermanabal@sadaf.org.ar> 